which it serves. Let no one imagine that this is a simple task. The complexity of the relations between the different functions and faculties must not deter us from the task, and the gravity of the danger which threatens all alike may well be sufficient at last to initiate the effort.

In regard to the advancement of learning, the university occupies a place in the research front. If knowledge is to be available to serve the wartime needs of the nation no less than to solve the problems of peace, some means must be found of keeping an appropriate balance between fundamental and applied research, of providing for the following-up of advances in knowledge, and of diverting effort to fields neglected or holding up the general advance. All this must be done without endangering that freedom of investigation and spirit of unprejudiced quest for truth which lie at the root of all scientific advance. Particularly is it important to provide for investigations in the domain of human relations, where lack of knowledge is already handicapping our war effort in many fields.

The advancement of knowledge links itself naturally with the teaching functions of the university through the thorny problem of the relation of teaching and research. Here we may well have to review the whole question of technique in the light of recent criticism and discussion on the use of the lecture or the tutorial system. Similarly, we have to consider the efficiency of research at the universities, not merely in terms of the claims of teaching, tutorial or administrative duties, but also in regard to the provision of adequate assistance or services in the laboratories, so that the best use is made of the time of qualified research workers.

These are questions which, like those of professional training, may well occupy the attention of the universities themselves and also of the many professional associations of scientific workers and their fellows. We cannot expect in the stress and strain of war to find and apply a completely satisfactory solution to them all. We may indeed need to be content with eliminating major causes of inefficiency and securing a modicum of co-operation in the pursuit of knowledge where it is most required. If, however, scientific and professional workers, whether within the walls of a university or without, are zealous enough in cherishing a high ideal of the functions of a university and give their minds, even in war-time, to such problems as these, then at least we may be sure that the universities of Great Britain will carry undimmed through these dark days their power to inspire, and to provide the loyal and unselfish leaders the nation needs both now and in the days of reconstruction to come.

\title{
CANCER: THE ROLE OF SURGERY, RADIUM AND $X$-RAYS IN ITS TREATMENT
}

$\mathrm{T}$ $\mathrm{HE}$ treatment of cancer has occupied an increasingly important position in public thought during the last few years. The debate in Parliament on the Cancer Bill laid bare the scanty provisions for adequate treatment existing in many parts of Britain. The war crisis in September which temporarily curtailed medical facilities resulted in an insistent demand for the treatment of cancer, a demand not immediately heard for the treatment of most other diseases. One aspect of this interest is the difference of opinion as to the proper scope of radium treatment relative to other methods, a difference illustrated by recent and present correspondence in NATURE $(144,973 ; 1939 ; 145,151 ; 1940$; also p. 347 of this issue).

Before attempting an assessment of the present status of cancer treatment, it will be instructive to examine first the extent to which cancer is in fact being treated in Great Britain at the present time, and the means available. A recent Ministry of Health inquiry* showed that in a representative sample 27 per cent of all cases were actually treated by either surgery or radiation, including purely palliative treatment. Allowing for possible groups of cases not coming into the sample, it is safe to say that less than 30 per cent of all cases occurring receive treatment. As to the means available, there are only two by which successful cure of cancer can be achieved-surgical excision and radium or X-ray therapy.

*'Cancer: An Enquiry into the Extent to which Patients receive Treatment." Reports on Public Health and Medical Subjects, No. 89 (H.M. Stationery Office.) 
Surgery was first in the field, and indeed was for many years the only available method of treatment. The principle underlying its use is simplicity itself; extensive removal of the growth along with the whole of the affected part. The effective use of surgery in cancer dates back to about half a century ago. At that time surgical techniques were evolved which are still known by the names of their creators-Halstead, Kocher, Wertheim, and many more. The surgery of cancer is now relatively stable; its accomplishments are known, its limitations accepted.

In contrast to surgery, radium and $\mathrm{X}$-ray therapy are still young and developing branches of medical science. Although radium was discovered in 1898 and was applied to minor medical use shortly afterwards, it did not become an important means of treating cancer until after the War of 1914-18. In the decade 1920-1930 the significance of the work which was being done in three now famous radiation therapy centres-the Fondation Curie in Paris, the Radiumhemmet in Stockholm, and the Memorial Hospital in New York-was gradually realized. It was not, however, until about the end of that decade that the impact of these revolutionary methods became generally felt. In Great Britain one result was the establishment of the Radium Trust and the creation of the National Radium Commission Centres for the provision of efficient radium treatment. It is then no overstatement to say that this new medical specialty is a mere ten years old, and still in an evolutionary phase. This fact has three consequences. In the first place, it is only over a very limited field of use that methods of treatment are as yet sufficiently standardized to represent an established practice of modern medicine. In the second place, it is vital that its value be judged in terms of its capacity as known to-day, and not in terms of its accomplishments of even a brief five years ago. Thirdly, it holds considerable promise for the future.

To arrive at an objective analysis of the relative value of surgery and radiation it is worth while first to examine the nature of the 27 per cent of treated cases mentioned above. The striking fact which emerges from such a study is that treatment is limited to a comparatively small number of cancer types. Of the 27 per cent of all cases occurring which in fact received treatment, no less than three-quarters belonged to one or other of the following five cancer groups: cancers of the stomach, intestine and rectum; cancer of the breast; cancer of the skin, including genitalia; cancers of the mouth, tongue and lip ; cancers of the uterus and vagina.

Cancer of the stomach, intestine and rectum constitutes almost exactly 400 out of every 1,000 cancer deaths and represents one of the very commonest types of cancer found. In view of this, it is depressing to find that only 8 per cent of this group are seen sufficiently early to permit an attempt at cure. The treatment is almost exclusively surgical and radiation is as yet of little value. A generous estimate of the percentage cured would be 15 per cent of cases treated.

Cancer of the breast constitutes 200 cases per 1,000 of all cancer deaths occurring in women. It was found to have been treated in 68 per cent of cases. Breast cancer undoubtedly represents the field in which surgery has accomplished its greatest victories. It is still the treatment of choice except in those cases which are inoperable when first seen. In these, radiation does provide a useful alternative method. In many cases both surgery and X-ray therapy are combined with great advantage. A fair estimate of the surgical cure-rate is $25-30$ per cent of all cases operated on. In the really early cases a cure rate of up to 75 per cent can be obtained. Cancer of the breast should be looked upon, therefore, as a mainly surgical field.

Carcinoma of the skin represents only a small proportion of all the cases occurring. It can be treated by wide surgical excision, or it can be treated by radiation-either radium or X-rayswith equal or even greater certainty of cure and without the same degree of disfigurement ; indeed, often such a return to normal occurs that the site from which the disease was eradicated cannot be distinguished. An estimate of the cure-rate of true skin cancer (squamous- cell carcinoma), adequately treated by either method, may be given as about 75 per cent. The less serious form of skin cancer, known as rodent ulcer, if treated properly rarely proves fatal. This field is therefore one which can be equally called surgical or radiological, but the cosmetic advantage lies with radiotherapy.

Cancer of the mouth constitutes just under 100 cases per 1,000 of all cases occurring in men, and analysis showed that 71 per cent were treated. There is little doubt now that radiation, particularly radium, has become the treatment of choice in this disease. The majority of mouth eancers occur in parts where surgery is not practicable. Where excision is possible, for example, in some carcinomas of the tongue, surgical cure is 
infrequent and is attended with marked disability. Extension of the disease from the mouth to the glands in the neck may, however, be dealt with either surgically or radiologically. Many cases of mouth cancer come for treatment when the disease is already advanced, yet even so cure-rates of 25-30 per cent for all the cases treated have been reported by British radiation therapy centres. For the early case, the chance of cure is at least 50 per cent. The mainstay of treatment of mouth cancer must therefore be accepted as being radium therapy.

Cancer of the uterus (uterine cervix) represents 133 cases per 1,000 deaths from cancer in women. Before the days of radiation it was treated surgically. This has now been abandoned in all modern schools. The present situation has been described in a recent survey where it is stated that "radiation offers a prognosis some 10 per cent better plus an appreciable salvage of inoperable cases. . . . To-day the prognosis from good radiation therapy is a one in two chance of cure in an operable case, and a one in seven chance in an inoperable case, and in selected centres the prognosis is even better, the chances being two in three and one in four respectively". . The rapid transition from surgical to radiation treatment is largely due to the statistical findings of the Health Section of the League of Nations. This disease can therefore be listed as an exclusively radiation field.

These five diseases together account for 75 per cent of the treated cases. The remainder include cancers of the lung, œesophagus, bladder, ovary, larynx and a large variety of less common lesions. Talking in general terms, treatment by surgery and by radiation is probablyabout equally common, and radiation is showing curative possibilities in a number of lesions which are impossible of surgical approach.

We see, therefore, that in three out of the five main curable types of cancer considered, radiation now occupies a place so generally accepted that patients treated otherwise cannot be considered as receiving the maximum chance of cure.

The value of radium and $\mathrm{X}$-ray therapy is, however, not limited to the treatment of forms of cancer in which permanent cure is possible. It can serve two other quite important functions. In a wide group of malignant growths, which include the lympho-sarcomata, radiation can control the disease and maintain the patient in a fair state of health for long periods. Another way in which radiation is extensively used is to obtain palliation of advanced cancers where cure is impossible. These cancers include the late stages of all those types which are curable in the early stages by radiation or surgery and many more. The growth of the tumour can be arrested temporarily, with disappearance of the attendant pain and disability. Even although life is only prolonged for a short time the reduction in suffering is great. Surgery has contributed two useful measures to the palliation of cancer-gastrostomy and colostomy. Both these measures, by affording mechanical relief in incurable cases, prolong life and reduce suffering.

Much has been written on the dangers of radiation treatment, as in the past of the dangers of surgical treatment. Where the disease concerned is cancer, in which the mortality of untreated cases is 100 per cent, danger should obviously not be overstressed. In surgical techniques a certain 'operative mortality' is regarded as inevitable. In the use of radiation for the cure of cancer similar 'treatment risk' must be faced. In both surgery and radiotherapy this risk varies in direct ratio to the magnitude of the undertaking and in inverse ratio to the skill of the operator or therapist. While the immediate risk of death is lower with radiation treatment, a new risk has to be taken, namely, that of local necrosis due to the treatment. At times this is unavoidable, and necroses may be faced at the time of treatment as being the price of what is regarded as curative treatment. Such necroses can be repaired later by plastic surgery.

The nature of the action of radiation on malignant tissue is a totally different process from that of excision, and radiation should not be thought of as a refined form of cautery. While surgery seeks the eradication of the disease by wide excision, radiation has a selective action on malignant cells in those groups of cases where the radiotherapist claims success. In the epitheliomata and the sensitive sarcomata it is possible to give a dose of radiation which will destroy the malignant cells while doing only temporary harm to the surrounding normal tissues. The margin between lethal dosage to the malignant cell and the lethal dose of the normal tissue is, however, quite a narrow one. To permit the successful use of this differential sensitivity an expert training in radiotherapy is necessary, both to assess a suitable dosage and to choose the best means of delivering that dosage accurately to the tumour with a minimal radiation of healthy tissue.

Radiation has occasionally been stated to 
produce metastases and to 'stimulate' the growth of malignant cells. Neither of these statements can be substantiated.

In the development of radiotherapy a vast amount of experimental work is still necessary. At present the techniques employed are based on clinical experience, which is necessarily conservative. The choice, for example, of radium versus $\mathrm{X}$-rays depends on the mechanical applicability of either to any particular lesion rather than on any known advantage of wave-length. In accessible sites it is usual to choose radium, which remains the more valuable of the two methods. $\mathrm{X}$-rays are used in the radiation of deep-seated lesions and in widening the zone of tissue treated from a radium source.
Anot factor which requires investigation is the optı_.um overall time of radiation. At present the times used depend on clinical experience, and it is felt that other overall times or time splittings of dosage may be found in the experimental field which will alter considerably our methods of treatment.

While stressing the need for developmental work in radiation methods and the value of surgery and radiation in their respective fields, it is necessary to state that the greatest increase in the cure-rate in these fields would be obtained by a successful campaign to get patients in the early stage of their disease. A well-planned scheme of public education and clinics as envisaged in the Cancer Bill would facilitate this.

\section{RUTHERFORD}

\section{Rutherford}

Being the Life and Letters of the Rt. Hon. Lord Rutherford, O.M. By Dr. A. S. Eve. Pp. xvi+ $451+18$ plates. (Cambridge: At the University Press, 1939.) 21s, net.

PRo ROF. EVE'S account of the life of his old friend Lord Rutherford treats it in the perfectly straightforward manner which such a book should. He takes the reader steadily through the life from boyhood in New Zealand to the 1851 Exhibition at Cambridge, the chair at Montreal, the chair at Manchester, and the return to Cambridge. A great part of the history is given through the medium of Rutherford's letters, many of the earlier ones being those written to his future wife.

The order of presentation is rather strictly chronological, so that successive paragraphs may deal with entirely different subjects ; for example, they may consist in accounts of a public lecture, a holiday journey, the organization of a department, and a new scientific discovery. In describing the purely scientific work, Prof. Eve at intervals devotes a few pages to a non-technical explanation of its import, which should help the inexpert reader in understanding the steady progress of the discoveries in which Rutherford took the leading part. In fact the biography is cast mainly in the form of a chronicle, and this is exactly the form it should have. There will remain for some later historian, who will have gained the perspective and impartiality of time, the task of assessing the respective merits of the enormous contributions that Rutherford made to physics. Taken in conjunction with his published books and papers, the present biography will provide very complete documenta. tion for such a historian, and it should go a long way towards protecting Rutherford from the two dangers to which the lives of the great seem to be specially exposed, on one side the degeneration into a dreary hagiography, and on the other the psychological account of the (usually Freudian). thoughts which the biographer thinks that his subject ought to have been thinking.

With a biography, as with a portrait, the author is always faced with an almost insuperable difficulty, that of satisfying both those who knew the subject and those who did not. The first expect to be reminded at every turn of the living original, whereas the others, with no personal memories, have to build the whole image out of the author's own work. So it is natural to ask whether Prof. Eve has 'got it across'. He has certainly given himself the best chance of doing so by his copious quotations from the letters. These, be it said, are mostly quite ordinary letters, dealing with the happenings of Rutherford's life, such as those written to his family when on a visit to America, or those to friends on the Continent renewing relations after the War of 1914-18. They are good straightforward descriptions of events, not embellished into anything like belles-lettres-had they been so one would have doubted their authorship-and anyone can feel that he could almost have written them himself. But not quite, for there is a tremendous common sense about them and a vigour raised to high power, which is the characteristic that his intimates will most 